\title{
Communication and Behavior Change in Health Education Practice
}

Corey H Basch*

Department of Public Health, William Paterson University, USA

\section{Introduction}

When and under what circumstances is it okay to use communications to evoke fear or guilt with the intent of influencing (or manipulating) an individual or group of individuals to make a certain choice? The field of health education and health promotion largely burgeoned as a result of the shift that began during the middle of the 1900's from infectious to chronic illness as the major causes of morbidity, mortality and disability, because these chronic diseases were caused, to a significant degree, by voluntary behaviors (smoking, diet, physical activity, use of health services, etc.). Infectious diseases and injuries are also determined to a great extent by voluntary choices. The health-related lifestyle choices of individuals were identified by governmental agencies as prime targets for intervention.

Examples of some of the most coercive approaches are formal social sanctions, namely laws. When traveling in a motor vehicle, passengers must, by law, wear a safety belt and drivers must refrain from talking on a cell phone (without a hands free device), even if they would choose to do otherwise. These laws and laws in general, will only work if they are enforced and if they are acceptable to the majority of the public. There are many examples of laws that are ineffective or only partially effective (e.g., the 21 year old drinking age, hands-free cell phone use). For many health related behaviors, coercion through laws is not a tenable or acceptable approach.

Health education specialists, in contrast, typically use communication (among other strategies) to help consumers make informed lifestyle choices. Yet health educators are likely to be under pressure from funders to "get results" beyond informed decisions and be accountable to change behavior. As health educators strive to understand and influence consumer behavior it is important to reflect on whether and under what circumstances persuasive or manipulative communications may be warranted. This commentary briefly considers some of these issues, including nature of the audience, countering existing manipulation, doing no harm, and long-versus short-term benefits.

\section{Nature of the Audience}

Who is or is not capable of making and informed choice? There is little debate that young children or adolescents are not "capable" of making certain kinds of informed choices. Thus actions to "protect" them are warranted-paternalism. There is little debate about implementing actions to coerce parents to treat children in certain ways (e.g., use restraints in cars, etc.). But there is more debate about the decision-making capability of other groups. What about the individual with a cognitivedisability or with depression? What about the individual with a terminal disease? These are questions of ethics and judgment, not science. While efforts to persuade or manipulate children and adolescents to adopt and practice health enhancing behaviors are generally accepted, efforts to educate them to make informed choices are equally warranted.

\section{Countering Existing Manipulation}

To what extent are there efforts to influence the public toward the less healthful choice? Many different forms of persuasive and manipulative mass communications have powerful negative effects on population health. In too many cases, such communications do not help the public make informed decisions about health, but rather aim to manipulate and persuade consumers for the sake of profit and greed. In some contexts there are extreme forces within American society "pushing" consumers to make health-compromising choices. One example is the billions in advertising and marketing spent annually to increase the initiation and use of tobacco products. In these cases, an using persuasive or manipulative communications may be viewed as "leveling the playing field." An alternative approach is to educate consumers to recognize and resist manipulative communication. This is challenging given the resources typically available for education versus those invested in marketing, advertising and promotions. A related and very important point here is that, if there is to be any substantive change in the nature and scope of consumers' health related behaviors, it is essential to address the social-environmental forces, including communication campaigns, that attempt to shape the health of the nation in noxious ways.

\section{Do No Harm}

How much certainty is there that the actions advocated through communications would not cause harm? It is unfortunate that public health actions throughout the relatively short U.S. history have sometimes resulted in unanticipated adverse effects. This undermines trust and compromises the potential effectiveness of subsequent communications. Examples from the mid-twentieth century include obtaining an annual chest X-ray or eating a diet rich in red meat, eggs and whole milk. Hormone replacement therapy is a more recent example. Clearly there are limits to science, which continue to be reflected today in controversies that confuse the public and create challenges for conceiving well-grounded public health communication campaigns. While persuading, manipulating or even coercing (e.g., through laws) the public to behave in ways that government agencies believe will improve the health status of the nation may be questioned in general, it is obviously particularly offensive when the action ultimately results in compromising public health.

\section{Long-Versus Short-term Benefits}

Does the individual's long-term autonomy justify short-term infringement? A reasonable person might agree that it is acceptable to manipulate or coerce someone, if that will result in increasing the

*Corresponding author: Corey $\mathrm{H}$ Basch, Assistant Professor of Public Health, Department of Public Health, William Paterson University, USA, Tel: 973-720-2603, E-mail: BASCHC@wpunj.edu

Received August 21, 2013; Accepted August 22, 2013; Published August 23 , 2013

Citation: Basch $\mathrm{CH}$ (2013) Communication and Behavior Change in Health Education Practice. J Mass Communicat Journalism 3: e139. doi:10.4172/21657912.1000e139

Copyright: (c) 2013 Basch $\mathrm{CH}$. This is an open-access article distributed under the terms of the Creative Commons Attribution License, which permits unrestricted use, distribution, and reproduction in any medium, provided the original author and source are credited. 
Citation: Basch CH (2013) Communication and Behavior Change in Health Education Practice. J Mass Communicat Journalism 3: e139. doi:10.4172/2165-7912.1000e139

Page 2 of 2

person's long-term liberty and autonomy. This argument is generally accepted when dealing with minors, but more likely to be questioned when the audience is adults. An associated issue is the certainty with which the long-term benefits will be realized. Can we guarantee that if an individual follows sound public health advice the result will be a long and fulfilling life? While the chances may be better for such outcomes, there is certainly nothing close to a guarantee. Indeed, what is a benefit itself is a value-laden issue. Virtually all public health interventions are justified, at least in part, on the basis of long term benefits, for example greater longevity. But what if the individual "decides" to live a life of health compromising behaviors, with a full understanding that the result is to jeopardize 10 or even 20 years of longevity? Is this the sign of an incompetent decision maker or a person that values some aspects of life more than others?

\section{Summary}

Health is a value-laden concept. The definition in policy and practice tends to follow a medical model and defines health in terms of the absence of disease. As such, public health policy and practice measures the effectiveness of investments based on traditional public health indices such as morbidity, mortality and disability. As health professionals, we should recognize that what we ask people to do may not always be reasonable from an individual's perspective, especially considering social, economic and environmental circumstances. Quality of life is increasingly being considered when establishing public health goals and policies, but it does not seem to carry as much weight as more traditional public health indices.

Perhaps the most important conclusion that can be drawn on this topic is that there has been and remains an emphasis on changing individuals' behaviors moreso than changing the social and environmental factors that foster the acquisition and maintenance of health compromising behaviors. When efforts are directed to influence the behavior of individuals, it is important for health education professionals to examine and establish a sound ethical basis for using manipulative or persuasive communications. Another worthy goal is to educate consumers to recognize and resist manipulative communications. 